\title{
Developing an Inpatient Group Psychotherapy Program: Challenges and Lessons Learnt
}

\author{
Emran Mohammad Razaghi, ${ }^{1}$ Maryam Tabatabaee, ${ }^{1}$ Ali Pourramzani, ${ }^{2, *}$ Reza Shirali \\ Mohammadpour, Arezou Mousazade Moghaddam, and Seyyed Taha Yahyavi, ${ }^{3,4}$ \\ ${ }_{1}^{1}$ Psychiatric and Clinical Psychology Research Center, Roozbeh Psychiatric Hospital, Tehran University of Medical Sciences, Tehran, IR Iran \\ ${ }_{2}^{2}$ Department of Psychiatry, School of Medicine, Shafa Hospital, Guilan University of Medical Sciences, Rasht, IR Iran \\ ${ }^{3}$ Department of Psychiatry, School of Medicine, Roozbeh Hospital, Tehran University Of Medical Sciences, Tehran, IR Iran \\ ${ }^{4}$ Psychiatry and Behavioral Sciences Research Center, Addiction institute, Mazandaran University of Medical Sciences, Sari, IR Iran \\ ${ }^{*}$ Correspanding author: Ali Pourramzani, Department of Psychiatry, School of Medicine, Shafa Hospital, Guilan University of Medical Sciences, Rasht, IR Iran. Tel: +98-1333666268, \\ Fax:+98-2155411811, E-mail: dr_pourramzani@yahoo.com
}

Received: August 8, 2014; Revised: October 6, 2014; Accepted: February 12, 2015

\begin{abstract}
In Iran, inpatient group psychotherapy has been limited to transient practices for research purposes or fulfilling personal interest of therapists. The goal of this paper is to share and explain the experience of developing an inpatient group psychotherapy program in Roozbeh Psychiatric Hospital, Tehran, Iran. After theoretical delineation and preparation of a draft of the program guideline, two pilot sessions were held. Based on this initial experience a final treatment guideline was prepared. Afterwards, the program was continued for more than 1 year in a female ward at Roozbeh Psychiatric Hospital. The output of this exercise was a guideline that covers important topics in development of inpatient group psychotherapy. It is concluded that inpatient group psychotherapy has its unique challenges. Of the most important challenges that can be mentioned in this regard are the participation of patients with significant differences in levels of psychopathology and psychiatric signs and symptoms, and high comorbidity with specific personality traits or disorders. Other challenges relevant to the structure of the group include items such as very limited time for working through and inevitable out-of-group contacts.
\end{abstract}

Keywords: Group Therapy; Inpatient; Psychiatry; Psychotherapy

\section{Background}

Group psychotherapy has been applied in clinical settings since about one hundred years ago (1). While the psychoanalytic approach was shadowed on individual and group psychotherapy in the first half of the twentieth century, other technics, especially, cognitive, existential, and interpersonal approaches, emerged and improved later (1).

The inpatient group psychotherapy method differs from its outpatient sibling in several aspects. Regarding group members' differences, hospitalized patients generally have more severe illnesses and are more likely to have a comorbid personality disorder (2). According to the limited duration of admission, the available time for inpatient psychotherapy is usually short (3). A third important difference is that in inpatient settings, the patients are living in a small common environment, thus the out-of-group communications and interactions are much more than what takes place in outpatient settings $(3,4)$. Psychotherapy is a complex process, and based on such differences, the inpatient variety is most likely even more complex.
Kosters et al. (2) evaluated the effectiveness of group psychotherapy in a meta-analysis of 70 published papers including 24 controlled and 46 pre- and post-measured studies, during the period between 1980 and 2004. Beneficial effects were found for the inpatient group therapy in controlled studies $(\mathrm{d}=0.31)$ and studies with pre- and post-data $(d=0.59)$. Several studies indicated the effectiveness of group therapy in severe psychiatric disorders like schizophrenia (5-7), bipolar disorder (8), and major depressive disorder (9). Yaeghoobi Nasrabadi et al. (9) conducted a controlled study comparing cognitive behavioral group therapy (CBGT) plus pharmacotherapy with pharmacotherapy alone in an inpatient setting for patients with major depressive disorder. They concluded that CBGT plus pharmacotherapy was significantly more effective than pharmacotherapy alone, especially in decreasing depression symptoms compared to anxiety $(\mathrm{P}<$ 0.05)(9).

Inpatient group psychotherapy is part of the standard treatment in many psychiatric wards around the world (10). In Iran, however, the condition has been somewhat

Copyright (C) 2015, Mazandaran University of Medical Sciences. This is an open-access article distributed under the terms of the Creative Commons Attribution-NonCommercial 4.0 International License (http://creativecommons.org/licenses/by-nc/4.0/) which permits copy and redistribute the material just in noncommercial usages, provided the original work is properly cited. 
different. Although, there have been several efforts to apply inpatient group psychotherapy, yet since most of them were based on individual interests or followed short-term research objectives $(9,11)$, they did not become a routine part of inpatient psychiatric treatment. For example, during the period of 1982-1985, Dr. Mahdi Ghazinoor practiced inpatient group psychotherapy at Roozbeh Psychiatric Hospital, Tehran, Iran. However, after his migration abroad, the program collapsed. The authors do not have detailed information about his work due to the lack of written reports. In 1986, Dr. Mohammad Sanati started inpatient groups at Roozbeh Psychiatric Hospital. In addition, in 1992 inpatient groups were held at Razi Psychiatric Hospital in the suburbs of Tehran under the supervision of Dr. Abdolhossein Rafatian. As mentioned, none of these efforts resulted in sustainable programs.

In May 2012, Dr. Ali Pourramzani and Dr. Reza Shirali-Mohammadpour, who were psychiatry residents at the time, became curious about inpatient group psychotherapy at Roozbeh Psychiatric Hospital (Tehran University of Medical Sciences, Tehran, Iran). Their objective was to explore potentials for further improvement of the quality of inpatient treatment, shortening hospitalization time, and fostering therapeutic alliance between inpatient clients. This paper is the abridged report of their experience, the challenges faced, and lessons learnt. As their approach has resulted in a sustainable program at Roozbeh Psychiatric Hospital, the authors hope that sharing their experience with other colleagues in Iran and elsewhere will help expand such measures as a routine part of inpatient psychiatric treatment.

\section{The Study Setting}

Roozbeh Psychiatric Hospital was the first modern psychiatric hospital in Iran, established in 1950 at its current location, and was affiliated to the Tehran University of Medical Sciences. Today, as an academic facility, Roozbeh Psychiatric Hospital is a center with 164 adult and 40 child psychiatry beds, and runs a psychiatric emergency ward with round the clock access and service with psychiatric and psychotherapeutic clinics active during the day. These services are covered by 26 faculty members and 48 residents of psychiatry. It is also an academic center for training medical students, psychiatric nurses, and masters of clinical psychology. In addition, residents of neurology and internal medicine spend their psychiatry rotation at Roozbeh Psychiatric Hospital. The hospital has two wards for females and four wards for males. Female ward number I (FWI), where our challenge took place, has 34 beds. Most of the admitted cases in this ward are patients with major psychiatric problems such as schizophrenia, mood disorders, and substance related disorders. The average length of hospitalization in this ward is about one month. The usual inpatient treatments include pharmacotherapy, electroconvulsive therapy(ECT), and recreational therapy.

\section{Developing the Idea}

The idea of applying inpatient group psychotherapy was first proposed by psychiatry residents who were eager to explore major psychiatric problems and their treatments more deeply, and to become more skilled psychiatrists in the future. The idea was welcomed by the faculty, and as the first step a scientific and an executive team was formed.

\section{Scientific and Executive Teams, and Their Roles and Duties}

The scientific team consisted of two psychiatry faculty members, two psychiatry residents, and one psychiatric nurse. The two residents were training for individual psychoanalytic psychotherapy and cognitive behavioral therapy. They had also participated as co-therapists or observers in outpatient group psychotherapy sessions for at least one year. The scientific team focused on the following tasks: a) review of related scientific material, b) preparation of the primary guideline, c) supervision of the pilot study, and d) revision of the guidelines for the final version.

The executive team consisted of two volunteer psychiatry residents, two volunteer psychiatric nurses, one social worker, the hospital manager, the hospital staff director, and the head nurse of FWI. They followed the task of preparation for the sessions, post-group supervision, and coordination with other relevant sections.

For improving the scientific basis of this project, a three-day workshop was held under the guidance of Dr. Masumeh Namavar (clinical psychologist, Cambridge University, Boston, USA), an experienced inpatient group psychotherapist. In the meantime, Dr. Irvin Yalom's "The Theory and Practice of Group Therapy" (12) was reviewed by the executive team. The scientific team searched the online literature by using the terms, inpatient and group psychotherapy as keywords. The scholarly papers were reviewed and used for planning of the pilot phase and writing the guidelines. Based on the literature review and discussions among the scientific team, it was decided that the general attitude of our inpatient group therapy would follow an interpersonal orientation with emphasis on here and now and problem solving.

Reference documents for the form and structure of the sessions were prepared. A final guideline was then developed by collating all documents. Group psychotherapy sessions were held in accordance with the preliminary version of the guideline. Next, in March 2012, two initial exploratory sessions were held. Furthermore, a final version of the guideline was prepared according to the findings and experiences of the two exploratory sessions. Subsequently, group sessions were held twice a week for more than one year until February 2014 (the duration covered by the current report). 


\section{The Guidelines}

Based on the findings and our experience throughout this process we made amendments and corrections to the guideline. The important parts of the final guideline were as follows:

Objective: the objective was to improve the quality of treatment, shorten the hospitalization period and strengthen the therapeutic alliance.

The psychotherapeutic approach: this approach was interpersonal with an emphasis on here and now and problem solving. Although the main approach of the group was interpersonal, the process of sessions was occasionally analyzed from a systemic and psychodynamic point of view for its educational benefits.

The structure of the group: the group structure was open, meaning that new cases could join the group at the start of any session, current members could leave the group upon discharge from the hospital, and attending group members could refuse to continue the group sessions for personal reasons.

Format of the sessions: sessions consisted of two circles; the inner circle with 12 patients and two co-therapists (a nurse and a resident of psychiatry) and the outer circle with observers and supervisors who did not interfere in the group process during the actual therapeutic session. Before and after each session, pre- and post-group supervision and discussions should be held. The optimal duration of therapeutic sessions was 45 minutes. Moreover, a pair of pre- and post-group supervision sessions of up to 30 minutes was found to be most helpful. A pregroup session would include information about the "to participate" group members. The co-therapists tried to align their methods and plans for the specific session. A discussion on the theme of the group conversation and the method of selection of the theme would also be held. In post-group sessions, aside from the scientific and executive team members, interested psychiatry residents and faculty members were also welcomed. A faculty member led the post-group session.

The time and place of sessions: the time and place of the sessions were fixed. The sessions started on time. After starting a session new patients were not allowed to join the group and if for any reason a patient would decide to leave the group, she was not permitted to rejoin the same session.

Regulations: group sessions were opened with a review of the regulations. Having respect for the other group members, in terms of their rights and dignity, was the first rule of the group. Another rule pointed to members' behavior; not speaking loudly or acting violently. In case of aggressive behavior or misconduct, the patient would be requested to leave the group. In the next step, participants and therapists introduced themselves by their names.

Participants: group participants were volunteer patients who had already been briefed by the ward nurses about the group process, and were offered and encouraged to participate in the specific group session. In addition, a written announcement was put on the ward board. Volunteer patients would then register for the group sessions. Patients with risk of aggressive behavior and/or highly irritable mood (e.g. patients experiencing severe manic episodes or withdrawal symptoms) would be excluded. With reference to evidence indicating that group therapy may improve the symptoms and signs of some patients that may not voluntarily participate in group sessions, such as patients with major depressive disorder or those with schizophrenia and a dominant negative feature, $(5,7)$ such patients were approached by the staff and were invited to participate in the group. Three out of 12 seats available during each session were reserved for these patients.

\section{Challenges and Lessons Learnt}

In the process of development of inpatient group psychotherapy, several lessons were learned:

Inpatient group psychotherapy is an effective therapy with intangible progress. As it is not yet defined as a routine inpatient psychiatric treatment in Iran, external incentives might help therapists endure its difficulties.

The attitude of supervisors and therapists directly influence the atmosphere of sessions. In the current experience, the two faculty members who supervised alternate sessions had somewhat different orientations. In fact, therapists were instructed to be more active and to emphasize on therapeutic elements such as universality, instillation of hope, and interpersonal learning in sessions ran under the supervision of one faculty member on Saturdays. However, on Tuesdays, when another faculty member supervised the group session, therapists were taught to be less active, and the emphasis was on dynamic therapeutic elements such as corrective recapitulation of the primary family group, catharsis and cohesiveness. The optimal time of sessions were found to be between 40 to 60 minutes. In more brief sessions, the group dynamic would not form, and in longer sessions, the group would become boring for patients who were experiencing major psychiatric problems. Under the experienced circumstances, we found that if the sessions were held between 1 and $2 \mathrm{pm}$, they would neither interfere with other daily therapeutic activities of the ward such as ECT doctors' visits, and recreational therapy sessions, nor with the patients' free time in the afternoon.

Despite the severe psychopathology of inpatient clients, psychodynamic phenomena, such as transference, countertransference, identification, and resistance were clearly observed.

In our setting, the head nurse was apparently a caretaker with close connection with patients of the ward during their stay. We found that patients identified the role of the head nurse in inpatient group therapy as unique; as a symbol of power, authority and parenthood. 
The different sitting positions of the two co-therapists within the group were examined. Having in mind that the group members in an inpatient setting generally have major psychiatric problems, it seems that if the two co-therapists sit opposite each other, they will have more control and influence on group processes.

Psychotic symptoms, such as delusions and hallucinations, appeared to be unimportant obstacles for the group process. On the contrary, we found that focusing some group discussions on such psychotic symptoms would have the potential to shed insight on these symptoms or relieve the patient from tensions.

Out of group interactions is an inevitable process of inpatient settings. While we were not happy with this dynamic at the beginning, our findings revealed that it could extend the therapeutic process of the group to ward interactions in the out-of-group hours. Therefore, it could simultaneously be regarded as an opportunity and a threat; an opportunity for further extension of therapeutic functions of the group session to ward interactions, and a threat to the confidentiality of group discussions. Nevertheless, repeating the group rules at the beginning of each session and emphasizing the importance of confidentiality might reduce this risk.

Evaluation of the effectiveness of inpatient group psychotherapy is difficult. As our groups were open and new members joined regularly and others left at a very fast pace and some patients only had the opportunity to participate in one session, the qualitative analysis of inpatient group psychotherapy may be more effective. It is noteworthy that according to our observations, each session has its specific dynamic effects. Qualitative-wise, our initiative appeared to be effective as patients and therapists observed and stated. However, the quantitative evaluation of inpatient group therapy remains a challenge. The authors are planning to make a valid, reliable, and culture consistent evaluation in the future.

While the authors hope to report specific categories of their initiative in a separate paper, they would welcome suggestions and comments by colleagues.

\section{Acknowledgements}

This study was supported by a grant from the Tehran University of Medical Sciences. This study was derived from Ali Pourramzani's postgraduate thesis in psychiatry. The authors would like thank the chief, manager, nurses, and staff of the Roozbeh Psychiatric Hospital, Tehran, IR Iran for their contribution towards conducting this study.

\section{Authors' Contribution}

Emran Mohammad Razaghi, Maryam Tabatabaee and Ali Pourramzani conceived and designed the evaluation, interpreted the clinical data, and critically revised the manuscript for important intellectual content. Reza Shirali Mohammadpour and Arezou Mousazade Moghaddam collected the clinical data. Seyyed Taha Yahyavi drafted and critically revised the manuscript for important intellectual content. All authors read and approved the final manuscript.

\section{Declaration of Interest}

None declared.

\section{References}

1. Spitz HI. Kaplan \& Sadock's concise textbook of clinical psychiatry New York: Lippincott Williams \& Wilkins; 2008. p. 682.

2. Kosters M, Burlingame GM, Nachtigall C, Strauss B. A meta-analytic review of the effectiveness of inpatient group psychotherapy. Group Dyn Theor Res. 2006;10(2):146-63.

3. Yalom ID. Inpatient group psychotherapy. New York: Basic Books; 1983.

4. Vinogradov S, Yalom ID. Concise guide to group psychotherapy. Arlington: American Psychiatric Pub;1989.

5. Gabrovsek VP. Inpatient group therapy of patients with schizophrenia. Psychiatr Danub. 2009;21 Suppl 1:67-72.

6. Kanas N. Inpatient and outpatient group therapy for schizophrenic patients. Am J Psychother. 1985;39(3):431-9.

7. Kanas N. Group Therapy for Schizophrenic Patients.Arlington: American Psychiatric Press; 1996. p. 157.

8. Weiss RD, Griffin ML, Greenfield SF, Najavits LM, Wyner D, Soto JA, et al. Group therapy for patients with bipolar disorder and substance dependence: results of a pilot study. J Clin Psychiatry. 2000;61(5):361-7.

9. Yaeghoobi Nasrabadi M, Atefvahid M, Ahmadzadeh G. The efficacy of cognitive-behavioral group therapy in reducing the level of depression and anxiety in patients with mood disorder. Iran J Psychiatry Clin Psychol. 2003;9(2):56-63.

10. Farley PN. Current practices in general hospital group psychotherapy. Blacksburg: Virginia Polytechnic Institute and State University; 1998.

11. Sanayi B, Nasiri H. Evaluation the effect of cognitive spiritual group therapy on decreasing anxiety and depression in mood disorder inpatients, Noor medical centre, Isfahan. Couns Res Dev. 2014;2(8):89-96.

12. Yalom ID, Leszcz M. The theory and practice of group psychotherapy. 4th ed. New York: Basic Books; 1995. 